\title{
Network Pharmacology-Based Investigation into the Mechanisms of Qibangyishen Formula for the Treatment of Diabetic Kidney Disease
}

\section{Zhi Wang}

Shanghai University of Traditional Chinese Medicine

\section{Guihua Jian ( $\nabla$ gh238@qq.com )}

Shanghai 6th Peoples Hospital Affiliated to Shanghai Jiaotong University School of Medicine https://orcid.org/0000-0001-5332-7638

\section{Research}

Keywords: Qibangyishen formula, Diabetic kidney disease, Network Pharmacology, Signaling pathways

Posted Date: February 9th, 2021

DOI: https://doi.org/10.21203/rs.3.rs-174220/v1

License: (c) This work is licensed under a Creative Commons Attribution 4.0 International License.

Read Full License 


\section{Abstract}

Background Diabetic kidney disease is the main microvascular complication of diabetes, and new treatment strategies are needed. We investigated the multi-component, multi-target, and multi-path mechanism of Qibangyishen formula for the treatment of diabetic kidney disease by network pharmacology methods.

Methods We collected the active ingredients and targets of Qibangyishen formula and examined diabetic kidney disease-related genes by searching the ETCM, TCMID, and DisGeNet databases. We constructed and analyzed the genetic network through Cytoscape software and used the STRING platform for pathway enrichment analysis.

Results We uncovered 421 active ingredients of Qibangyishen formula, corresponding to 748 targets. A network analysis screen revealed 47 hub-node targets, and 13 of these targets were diabetic kidney disease-related disease genes. Further functional analysis identified 26 targets acting on 11 pathways related to the development of diabetic kidney disease, including PI3K-Akt, thyroid hormone, neurotrophin, Wnt, chemokine, and osteoclast differentiation signaling pathways.

Conclusions This study suggests that Qibangyishen formula regulates multiple genes and biological processes related to diabetic kidney disease and provides a foundation for further research and clinical applications.

\section{Background}

Diabetic kidney disease (DKD) is a chronic disease caused by diabetes and has a complicated pathogenesis [1-3]. The clinical features include continuously increasing excretion of albuminuria and/or glomerular filtration rate (GFR) less than $60 \mathrm{ml} /\left(\min \cdot 1.73 \mathrm{~m}^{2}\right)$ for more than 3 months, which can eventually develop into end-stage renal disease (ESRD). Diabetic kidney disease leads to about $30-50 \%$ ESRD cases worldwide $[4,5]$ and is the leading cause of ESRD in middle-aged and elderly people in China $[6,7]$.

There are many clinical treatments for diabetic kidney disease $[2,8,9]$, including life management [10, $11]$, blood sugar control [12, 13], blood pressure control [14, 15], blood lipid regulation [16, 17], uric acid control [18, 19], and proteinuria regulation [20]. These treatments have various degrees of efficacy, but many patients with DKD continue to progress to dialysis. Traditional Chinese medicine has also been widely used to treat diabetic kidney disease. Chinese medicine may improve kidney and immune function and elicit therapeutic effects [21-23]. Diabetic kidney disease has no precise definition in ancient Chinese medical literature, but according to etiology and pathogenesis, diabetic kidney disease can be attributed to "edema" and "guange" secondary to Xiaoke. Huang et al. [24] found that QufengTongluo Formula and YiqiHuayu Formula delay renal fibrosis and reduce urine protein in DKD rats, which may be related to enhanced PTEN expression, inhibition of PI3K/Akt signaling, and reduced transforming growth factor- $\beta$ (TGF- $\beta$ ) expression. Hong et al. [25] showed that Jiangtang Formula reduces the level of advanced 
glycosylation products in KK-Ay mice and increases the phosphorylation of PI3K and Akt, which reduces inflammatory responses in DKD rats.

Qibangyishen Formula (QBYSF) is composed of DI HUANG (Rehmannia glutinosa Libosch.), HUANG QI (Astragalus membranaceus(Fisch.)Bge.), SAN QI (Panax notoginseng(Burk.)F. H. Chen), NIU BANG ZI (Arctium lappa L.), BAI SHAO (Paeonia lactiflora Pall.) and HEI DOU (Glycine max(L.)Merr.). QBYSF has been clinically used to treat diabetic kidney disease, and ingredients within QBYSF have been shown to have beneficial effects. Specifically, DI HUANG reduces blood sugar, serum triglycerides, uric acid, and the 24-hour urine albumin excretion rate, increases high density lipoprotein, interferes with inflammation, significantly improves serum creatinine and urea nitrogen, and protects patients' renal function [26, 27]. HUANG QI reduces blood sugar, uric acid, urea nitrogen, and blood creatinine, improves proteinuria, and has a protective effect on the kidneys [28-30]. SAN QI regulates sugar metabolism, has anti-fibrosis, antioxidation and anti-inflammatory effects, improves kidney function, and delays kidney damage [31, 32]. Previously, we found that HUANG QI combined with SAN QI reduces urinary protein excretion, decreases renal pathological changes, and improves apoptosis and shedding of kidney podocytes in DKD rats [33]. NIU BANG ZI extract reduces podocyte damage and proteinuria symptoms of DKD mice by enhancing PP2A activity in podocytes and protects kidney function [34].NIU BANG ZI extract also significantly reduces blood sugar in DKD mice and reduces the damage to the kidney [35]. Total glycosides is the main component of the BAI SHAO and reduces serum hs-CRP, TNF- $a$, and MCP- 1 levels in patients with DKD, thereby protecting the kidneys [36]. Peony total glucosides prevent renal tubular interstitial damage in rats with diabetic nephropathy and delay the development of diabetic nephropathy [37]. HEI DOU reduces blood sugar, blood lipids, serum creatinine, and blood urea nitrogen in streptozotocin (STZ)-induced diabetic rats [38], and long-term low-dose HEI DOU protects diabetic renal insufficiency [39]. Although, the effects of individual components of QBYSF have been examined in diabetic murine models and in patients, the mechanism of QBYSF on diabetic kidney disease is not clear yet.

This study uses network pharmacology to screen the main active ingredients, targets, and pathways of QBYSF to analyze its mechanism on the treatment of diabetic kidney disease.

\section{Materials And Methods}

\section{Collection of active ingredients and potential targets of QBYSF.}

QBYSF is composed of six herbs: DI HUANG (Rehmannia glutinosa Libosch.), HUANG QI (Astragalus membranaceus(Fisch.)Bge.), SAN QI (Panax notoginseng(Burk.)F. H. Chen), NIU BANG ZI (Arctium lappa L.), BAI SHAO (Paeonia lactiflora Pall.) and HEI DOU (Glycine max(L.)Merr.).Based on the pinyin name of each herb, we searched ETCM [40] and TCMID [41] databases to identify the active ingredients of QBYSF. In addition, we searched the ETCM database for the names of traditional Chinese medicines and compounds that are potential targets of the compounds contained in QBYSF. 


\section{Collection of targets for diabetic kidney disease.}

Using "Diabetic Kidney Disease" as a keyword, we searched DisGeNet [42] to find genes and targets related to diabetic kidney disease.

\section{Constructing a sub-network affected by diabetic kidney disease.}

In November 2019, we downloaded all high-quality binary protein-protein interactions from a high quality proteomics database HINT (High-quality INTeractomes) [43] and used Cytoscape [44] software to construct a genome-wide protein-protein interaction network in humans. This network is named the HINT network, which contains 12933 nodes and 60672 edges. We mapped all disease genes from diabetic kidney disease to the HINT network and extracted all nodes, neighbor nodes, and edges between all nodes related to diabetic kidney disease, but only retained the largest connected components to construct the diabetic kidney disease sub-network.

\section{Constructing an association network between compound herbal medicines and important targets.}

We used Cytoscape software to perform topology analysis on the diabetic kidney disease sub-network. We calculated the degree for each node and kept all nodes with a degree greater than twice the average degree for the network's hub nodes. The targets corresponding to the hub nodes were defined as important targets of QBYSF for the treatment of diabetic kidney disease. Cytoscape software was used to construct an association network between targets and corresponding herbs.

\section{Pathway enrichment analysis}

We entered the target gene ID into the STRING [45] platform for pathway enrichment analysis.

\section{Results}

\section{Active ingredients and potential targets of QBYSF.}

In the ETCM and TCMID databases, the herbs of QBYSF were searched, and 421 active ingredients of QBYSF were obtained (Supplementary Table 1), corresponding to 748 targets (Supplementary Table 2). The number of compounds and targets of each herbal medicine is shown in Table 1, and the intersection between herbal targets is shown in Fig. 1A. 
Table 1

The number of compounds and corresponding potential targets contained in the herbal medicine of QBYSF

\begin{tabular}{|llll|}
\hline Herb Latin name & $\begin{array}{l}\text { Herb Pinyin } \\
\text { name }\end{array}$ & $\begin{array}{l}\text { Number of } \\
\text { compounds }\end{array}$ & $\begin{array}{l}\text { Number of } \\
\text { targets }\end{array}$ \\
\hline Rehmannia glutinosa Libosch. & DI HUANG & 67 & 393 \\
\hline $\begin{array}{l}\text { Astragalus } \\
\text { membranaceus(Fisch.)Bge. }\end{array}$ & HUANG QI & 73 & 557 \\
\hline Panax notoginseng(Burk.)F. H. Chen & SAN QI & 168 & 290 \\
\hline Arctium lappa L. & NIU BANG ZI & 28 & 93 \\
\hline Paeonia lactiflora Pall. & BAI SHAO & 67 & 345 \\
\hline Glycine max(L.)Merr. & HEI DOU & 43 & 257 \\
\hline
\end{tabular}

\section{Genes related to diabetic kidney disease.}

We searched the DisGeNet database to identify diabetic kidney disease-related target genes. We obtained 559 diabetic kidney disease-related genes (Supplementary Table 3), of which 66 disease genes were potentially targeted by QBYSF (Fig. 1B).

\section{The diabetic kidney disease sub-network.}

The diabetic kidney disease sub-network had 3467 nodes and 23661 edges and included genes related to diabetic kidney disease and neighboring genes in the human protein-protein interaction network. We mapped QBYSF targets to this network, identified 219 targets, and found 52 related to diabetic kidney disease (Fig. 2).

\section{Identification of QBYSF targets.}

We calculated the degree for each node in the diabetic kidney disease sub-network, and the average degree for the network was 13.6. The nodes with a degree greater than 27 were labeled hub nodes for the sub-network. There were 444 hub nodes in total, 47 were QBYSF targets, and 13 QBYSF targets were related to diabetic kidney disease.

The correlation network between the 47 QBYSF targets and QBYSF ingredient herbs is shown in Fig. 3A. HUANG QI (Astragalus membranaceus(Fisch.)Bge.) and DI HUANG (Rehmannia glutinosa Libosch.) had many common targets, and BAI SHAO (Paeonia lactiflora Pall.), NIU BANG ZI (Arctium lappa L.), SAN QI (Panax notoginseng(Burk.)F. H. Chen), and the adjuvant HEI DOU (Glycine max(L.)Merr.) also shared many common targets. 
In general, a natural compound's effect on a target is lower than that of a drug because a drug's structure is designed to increase its binding affinity to the target. Although a single natural compound may have small effect on a target in isolation, targeting the same protein by multiple compounds by multiple compounds may synergistically increase the effect on the target, thereby producing a therapeutic effect. We defined a protein target score by the number of herb-compound pairs that act on a protein. There were 43 of 47 QBYSF targets with a score equal or greater than two, which suggests that QBYSF may regulate these targets.

\section{Enrichment analysis of QBYSF targets}

We examined 219 QBYSF targets including the 47 hub-node targets in the diabetic kidney disease subnetwork using the STRING platform for functional enrichment analysis. We used an FDR $<0.0001$ to identify KEGG pathways significantly enriched in QBYSF targets and selected the top 20 signal pathways significantly regulated by QBYSF (Figure 4).

The signal pathways regulated by the two target sets include PI3K-Akt, thyroid hormone, neurotrophin, and osteoclast differentiation signal pathways (Figure 4A, B). The intersection of the two sets in Figure 4A and 4B includes 11 signal pathways, which include 26 hub-node targets (Figure 5).

\section{Discussion}

The main characteristics of Chinese medicine are a "holistic concept" and "symptom differentiation and treatment", and network pharmacology research methods are similar to this "holistic concept". Network pharmacology for Chinese medicine is an emerging and rapidly developing cross-disciplinary field, and includes databases, network servers, and software to study TCM network pharmacology $[46,47]$.

In this study, we used network pharmacology to study the mechanism of Qibangyishen formula for the treatment of diabetic kidney disease. Based on TCM databases, 421 active ingredients in QBYSF were identified, corresponding to 748 targets. There were 559 diabetic kidney disease-related genes obtained from the DisGeNet database. Network analysis revealed 47 QBYSF targets, and 13 of these were diabetic kidney disease-related disease genes. Pathway enrichment analysis found that 26 of the 47 targets were regulated in 11 pathways related to endocrine and renal functions, including PI3K-Akt signaling, thyroid hormone signaling, estrogen signaling, insulin signaling, osteoclast differentiation, and Wnt signaling.

The phosphatidylinositol 3'-kinase (PI3K)-protein kinase B (Akt) signaling pathway regulates the cell cycle, transcription, translation, proliferation, growth, and survival in response to extracellular signals such as toxins. Abnormal PI3K/AKT signaling can cause cancer [48-50], diabetes [51-53], and other diseases. PI3K-Akt signaling is closely related to the pathogenesis of DKD and is a common target in many traditional Chinese medicine compounds. Huang et al. [24] found that enhancing the expression of PTEN, inhibiting the PI3K/Akt signaling pathway, and reducing TGF- $\beta$ expression can delay renal fibrosis and reduce urine protein in DKD rats. Hong et al. [25] showed that the Chinese medicine Jiangtang 
reduces the level of advanced glycosylation products in KK-Ay mice and increases the phosphorylation of $\mathrm{PI3K}$ and Akt, thereby reducing the inflammatory response of DKD. Increased activation of PI3K-Akt signaling can also aggravate Tubulointerstitial Fibrosis (TIF) [54].

The Wnt signaling pathway, highly conserved in various species, is a signal transduction pathway that includes proteins that pass signals through cell surface receptors, and is essential in developmental processes. Continuous activation of Wnt signaling induces fibroblast proliferation, which can lead to renal fibrosis, which can then be reversed by inhibiting Wnt signaling [55]. Wnt signaling triggers the pathogenesis of podocyte dysfunction and proteinuria [56-58]. Targeted inhibition of Wnt / $\beta$-catenin signaling transmission can preserve podocyte integrity and is used in animal models $[59,60]$. Diabetes can cause overactivation of Wnt1/ $\beta$-catenin signaling, induces renal fibrosis and podocyte damage, and inhibition of Wnt1/ $\beta$-catenin signaling improves DKD progression $[61,62]$.

Insulin is the only hormone in the body that lowers blood sugar. Inadequate insulin secretion causes high blood sugar and may lead to diabetes. The regulatory effect of insulin on metabolism is mainly mediated by the PI3K signaling pathway [63]. Podocyte insulin sensitivity is critical to renal function, and dysregulated podocyte insulin response can result in podocyte damage [64]. Intracellular lipids are the main determinants of podocyte insulin signal transduction and contributes to the pathogenesis of DKD [65]. Sadi et al. found that repairing the components of the insulin signaling pathway improves kidney oxidative stress and inflammation caused by diabetes [66].

Thyroid hormones are produced and released by the thyroid gland. Thyroid hormones have an important role in kidney physiology, which is manifested in the growth, development, and maintenance of kidney homeostasis $[67,68]$. Chemokines also affect DKD, and inhibiting chemokine secretion has antiinflammatory and anti-fibrotic effects, which delays DKD progression [69].

\section{Conclusion}

In summary, our network pharmacological study suggests that QBYSF protects the kidneys through 26 targets that regulate 11 pathways involved in the progression of diabetic kidney disease, including PI3KAkt signaling, Wnt signaling, insulin signaling, and chemokine signaling, which delays the progression of diabetic kidney disease.

\section{Abbreviations}

DKD: Diabetic kidney disease; GFR: Glomerular Filtration Rate; ESRD: End-Stage Eenal Disease; QBYSF: Qibangyishen Formula; HINT: High-quality INTeractomes; TIF: Tubulointerstitial Fibrosis.

\section{Declarations}

\section{Ethics approval and consent to participate}




\section{Consent to publish}

Not applicable

\section{Availability of data and materials}

Relevant data associated with the study are available in the Supplementary Materials of this paper.

\section{Competing interests}

The authors declare that they have no competing financial interests.

\section{Funding}

This work was sponsored by the Clinical and Experimental Research of YSHS Granule.

\section{Authors' Contributions}

Zhi Wang, Guihua Jian: conception and design of the study, and generation and collection of data. Wang Zhi: assembly, analysis, and interpretation of data, drafting and revision of the manuscript. All authors approved of the final version of the manuscript.

\section{Acknowledgements}

Not applicable

\section{References}

1. Kanwar YS, Sun L, Xie P, Liu FY, Chen S (2011) A glimpse of various pathogenetic mechanisms of diabetic nephropathy. Annu Rev Pathol 6:395-423. doi:10.1146/annurev.pathol.4.110807.092150

2. Thomas MC, Brownlee M, Susztak K, Sharma K, Jandeleit-Dahm KA, Zoungas S, Rossing P, Groop PH, Cooper ME (2015) Diabetic kidney disease. Nat Rev Dis Primers 1:15018.

doi:10.1038/nrdp.2015.18

3. Wei L, Xiao Y, Li L, Xiong X, Han Y, Zhu X, Sun L (2018) The Susceptibility Genes in Diabetic Nephropathy. Kidney Dis (Basel) 4 (4):226-237. doi:10.1159/000492633 
4. Ruiz-Ortega M, Rodrigues-Diez RR, Lavoz C, Rayego-Mateos S (2020) Special Issue "Diabetic Nephropathy: Diagnosis, Prevention and Treatment". J Clin Med 9 (3). doi:10.3390/jcm9030813

5. Tuttle K, Bakris G, Bilous R, Chiang J, de Boer I, Goldstein-Fuchs J, Hirsch I, Kalantar-Zadeh K, Narva A, Navaneethan S, Neumiller J, Patel U, Ratner R, Whaley-Connell A, Molitch M (2014) Diabetic kidney disease: a report from an ADA Consensus Conference. Diabetes care 37 (10):2864-2883. doi:10.2337/dc14-1296

6. Hou JH, Zhu HX, Zhou ML, Le WB, Zeng CH, Liang SS, Xu F, Liang DD, Shao SJ, Liu Y, Liu ZH (2018) Changes in the Spectrum of Kidney Diseases: An Analysis of 40,759 Biopsy-Proven Cases from 2003 to 2014 in China. Kidney Dis (Basel) 4 (1):10-19. doi:10.1159/000484717

7. Zhang Q, Ren H, Xie J, Li X, Huang X, Chen N (2014) Causes of death in peritoneal dialysis patients with different kidney diseases and comorbidities: a retrospective clinical analysis in a Chinese center. International urology and nephrology 46 (6):1201-1207. doi:10.1007/s11255-013-0561-5

8. National Kidney F (2012) KDOQI Clinical Practice Guideline for Diabetes and CKD: 2012 Update. American journal of kidney diseases : the official journal of the National Kidney Foundation 60 (5):850-886. doi:10.1053/j.ajkd.2012.07.005

9. Barrera-Chimal J, Jaisser F (2020) Pathophysiologic mechanisms in diabetic kidney disease: A focus on current and future therapeutic targets. Diabetes, obesity \& metabolism:16-31. doi:10.1111/dom.13969

10. Cupisti A, Brunori G, Di lorio BR, D'Alessandro C, Pasticci F, Cosola C, Bellizzi V, Bolasco P, Capitanini A, Fantuzzi AL, Gennari A, Piccoli GB, Quintaliani G, Salomone M, Sandrini M, Santoro D, Babini P, Fiaccadori E, Gambaro G, Garibotto G, Gregorini M, Mandreoli M, Minutolo R, Cancarini G, Conte G, Locatelli F, Gesualdo L (2018) Nutritional treatment of advanced CKD: twenty consensus statements. J Nephrol 31 (4):457-473. doi:10.1007/s40620-018-0497-z

11. American Diabetes A (2020) Diabetes Advocacy: Standards of Medical Care in Diabetes-2019. Diabetes Care 43 (Suppl 1):S203-S204. doi:10.2337/dc20-S016

12. Coresh J, Turin TC, Matsushita K, Sang Y, Ballew SH, Appel LJ, Arima H, Chadban SJ, Cirillo M, Djurdjev O, Green JA, Heine GH, Inker LA, Irie F, Ishani A, Ix JH, Kovesdy CP, Marks A, Ohkubo T, Shalev V, Shankar A, Wen CP, de Jong PE, Iseki K, Stengel B, Gansevoort RT, Levey AS (2014) Decline in estimated glomerular filtration rate and subsequent risk of end-stage renal disease and mortality. JAMA 311 (24):2518-2531. doi:10.1001/jama.2014.6634

13. Wong MG, Perkovic V, Chalmers J, Woodward M, Li Q, Cooper ME, Hamet P, Harrap S, Heller S, MacMahon S, Mancia G, Marre M, Matthews D, Neal B, Poulter N, Rodgers A, Williams B, Zoungas S, Group A-OC (2016) Long-term Benefits of Intensive Glucose Control for Preventing End-Stage Kidney Disease: ADVANCE-ON. Diabetes Care 39 (5):694-700. doi:10.2337/dc15-2322

14. Adler Al, Stevens RJ, Manley SE, Bilous RW, Cull CA, Holman RR (2003) Development and progression of nephropathy in type 2 diabetes: the United Kingdom Prospective Diabetes Study (UKPDS 64). Kidney international 63 (1):225-232 
15. Emdin CA, Rahimi K, Neal B, Callender T, Perkovic V, Patel A (2015) Blood pressure lowering in type 2 diabetes: a systematic review and meta-analysis. JAMA 313 (6):603-615.

doi:10.1001/jama.2014.18574

16. Catapano AL, Graham I, De Backer G, Wiklund O, Chapman MJ, Drexel H, Hoes AW, Jennings CS, Landmesser U, Pedersen TR, Reiner Z, Riccardi G, Taskinen MR, Tokgozoglu L, Monique Verschuren WM, Vlachopoulos C, Wood DA, Luis Zamorano J, Additional C, Cooney MT (2017) 2016 ESC/EAS Guidelines for the Management of Dyslipidaemias. Rev Esp Cardiol (Engl Ed) 70 (2):115. doi:10.1016/j.rec.2017.01.002

17. Grundy SM, Stone NJ, Bailey AL, Beam C, Birtcher KK, Blumenthal RS, Braun LT, de Ferranti S, FaiellaTommasino J, Forman DE, Goldberg R, Heidenreich PA, Hlatky MA, Jones DW, Lloyd-Jones D, LopezPajares N, Ndumele CE, Orringer CE, Peralta CA, Saseen JJ, Smith SC, Jr., Sperling L, Virani SS, Yeboah J (2019) 2018 AHA/ACC/AACVPR/AAPA/ABC/ACPM/ADA/AGS/APhA/ASPC/NLA/PCNA Guideline on the Management of Blood Cholesterol: A Report of the American College of Cardiology/American Heart Association Task Force on Clinical Practice Guidelines. J Am Coll Cardiol 73 (24):e285-e350. doi:10.1016/j.jacc.2018.11.003

18. Mauer M, Doria A (2020) Uric acid and risk of diabetic kidney disease. Journal of nephrology. doi:10.1007/s40620-020-00796-z

19. Feig D (2020) Urate-Lowering Therapy and Chronic Kidney Disease Progression. The New England journal of medicine 382 (26):2567-2568. doi:10.1056/NEJMe2015886

20. Araki S-i, Haneda M, Koya D, Hidaka H, Sugimoto T, Isono M, Isshiki K, Chin-Kanasaki M, Uzu T, Kashiwagi A (2007) Reduction in microalbuminuria as an integrated indicator for renal and cardiovascular risk reduction in patients with type 2 diabetes. Diabetes 56 (6):1727-1730

21. Chen H, Pan H, Chen Y, Chen Y, Lin Y, Yang S, Chen J, Wu H (2019) Traditional Chinese medicine use is associated with lower end-stage renal disease and mortality rates among patients with diabetic nephropathy: a population-based cohort study. BMC complementary and alternative medicine 19 (1):81. doi:10.1186/s12906-019-2491-y

22. Sun G, Li C, Cui W, Guo Q, Dong C, Zou H, Liu S, Dong W, Miao L (2016) Review of Herbal Traditional Chinese Medicine for the Treatment of Diabetic Nephropathy. Journal of diabetes research 2016:5749857. doi:10.1155/2016/5749857

23. Wen Y, Yan M, Zhang B, Li P (2017) Chinese medicine for diabetic kidney disease in China. Nephrology (Carlton, Vic):50-55. doi:10.1111/nep.13149

24. Wei-Jun H, Qiang F, Yong-Hua X, Qing G, Wen-Jing W, Zi-Long S, Hua Z, Xu J, Xue-Min H, Ya-Xin Z, JinXi Z, Shi-Dong W, Mian J, Yu-Ting Z (2018) Effect of Qufengtongluo Decoction on PI3K/Akt Signaling Pathway in the Kidney of Type 2 Diabetes Mellitus Rat (GK Rat) with Diabetic Nephropathy. Evidencebased complementary and alternative medicine : eCAM 2018

25. Hong JN, Li WW, Wang LL, Guo H, Jiang Y, Gao YJ, Tu PF, Wang XM (2017) Jiangtang decoction ameliorate diabetic nephropathy through the regulation of PI3K/Akt-mediated NF-kappaB pathways in KK-Ay mice. Chin Med 12:13. doi:10.1186/s13020-017-0134-0 
26. Xu Z, Dai X, Zhang Q, Su S, Yan H, Zhu Y, Shang E, Qian D, Duan J (2020) Protective effects and mechanisms of Rehmannia glutinosa leaves total glycoside on early kidney injury in $\mathrm{db} / \mathrm{db}$ mice. Biomedicine \& pharmacotherapy = Biomedecine \& pharmacotherapie 125:109926. doi:10.1016/j.biopha.2020.109926

27. Jiang P, Xiang L, Chen Z, Lu H, Zhou L, Yang L, Ji Y, Liu Y, Sun X, Deng Y, Nie X, Luo R, Zhao X (2018) Catalpol alleviates renal damage by improving lipid metabolism in diabetic $\mathrm{db} / \mathrm{db}$ mice. American journal of translational research 10 (6):1750-1761

28. Zhou X, Zou J, Ao C, Gong D, Chen X, Ma Y (2020) Renal protective effects of astragaloside IV, in diabetes mellitus kidney damage animal models: A systematic review, meta-analysis. Pharmacological research 160:105192. doi:10.1016/j.phrs.2020.105192

29. Zhang Y, Tao C, Xuan C, Jiang J, Cao W (2020) Transcriptomic Analysis Reveals the Protection of Astragaloside IV against Diabetic Nephropathy by Modulating Inflammation. Oxidative medicine and cellular longevity 2020:9542165. doi:10.1155/2020/9542165

30. Chen X, Wang H, Jiang M, Zhao J, Fan C, Wang Y, Peng W (2018) Huangqi (astragalus) decoction ameliorates diabetic nephropathy via IRS1-PI3K-GLUT signaling pathway. American journal of translational research 10 (8):2491-2501

31. Wang T, Guo R, Zhou G, Zhou X, Kou Z, Sui F, Li C, Tang L, Wang Z (2016) Traditional uses, botany, phytochemistry, pharmacology and toxicology of Panax notoginseng (Burk.) F.H. Chen: A review. Journal of ethnopharmacology 188:234-258. doi:10.1016/j.jep.2016.05.005

32. Du Y, Wang L, Qian J, Zhang K, Chai K (2016) Panax notoginseng saponins protect kidney from diabetes by up-regulating silent information regulator 1 and activating antioxidant proteins in rats. Chinese journal of integrative medicine 22 (12):910-917. doi:10.1007/s11655-015-2446-1

33. Zhai R, Jian G, Chen T, Xie L, Xue R, Gao C, Wang N, Xu Y, Gui D (2019) Astragalus membranaceus and Panax notoginseng, the Novel Renoprotective Compound, Synergistically Protect against Podocyte Injury in Streptozotocin-Induced Diabetic Rats. J Diabetes Res 2019:1602892. doi:10.1155/2019/1602892

34. Zhong Y, Lee K, Deng Y, Ma Y, Chen Y, Li X, Wei C, Yang S, Wang T, Wong N, Muwonge A, Azeloglu E, Zhang W, Das B, He J, Liu R (2019) Arctigenin attenuates diabetic kidney disease through the activation of PP2A in podocytes. Nature communications 10 (1):4523. doi:10.1038/s41467-01912433-w

35. Zhang J, Cao P, Gui J, Wang X, Han J, Wang Y, Wang G (2019) Arctigenin ameliorates renal impairment and inhibits endoplasmic reticulum stress in diabetic $\mathrm{db} / \mathrm{db}$ mice. Life sciences 223:194201. doi:10.1016/j.Ifs.2019.03.037

36. Zhu Q, Qi X, Wu Y, Wang K (2016) Clinical study of total glucosides of paeony for the treatment of diabetic kidney disease in patients with diabetes mellitus. International urology and nephrology 48 (11):1873-1880. doi:10.1007/s11255-016-1345-5

37. Zhang W, Zhao L, Su S, Xu X, Wu Y (2014) Total glucosides of paeony attenuate renal tubulointerstitial injury in STZ-induced diabetic rats: role of Toll-like receptor 2. Journal of 
pharmacological sciences 125 (1):59-67. doi:10.1254/jphs.13173fp

38. Qiu J, Zhu H, Liu P, Wang J, Lu L (2018) Protective effects of dietary polyphenols from black soybean seed coats on islet and renal function in streptozotocin-induced diabetic rats. Journal of the science of food and agriculture 98 (6):2350-2359. doi:10.1002/jsfa.8726

39. Chen Z, Wang C, Pan Y, Gao X, Chen H (2018) Hypoglycemic and hypolipidemic effects of anthocyanins extract from black soybean seed coat in high fat diet and streptozotocin-induced diabetic mice. Food \& function 9 (1):426-439. doi:10.1039/c7fo00983f

40. Xu H, Zhang Y, Liu Z, Chen T, Lv C, Tang S, Zhang X, Zhang W, Li Z, Zhou R, Yang H, Wang X, Huang L (2019) ETCM: an encyclopaedia of traditional Chinese medicine. Nucleic acids research 47:D976D982. doi:10.1093/nar/gky987

41. Xue R, Fang Z, Zhang M, Yi Z, Wen C, Shi T (2013) TCMID: Traditional Chinese Medicine integrative database for herb molecular mechanism analysis. Nucleic acids research 41:D1089-1095. doi:10.1093/nar/gks1100

42. Piñero J, Bravo À, Queralt-Rosinach N, Gutiérrez-Sacristán A, Deu-Pons J, Centeno E, García-García J, Sanz F, Furlong L (2017) DisGeNET: a comprehensive platform integrating information on human disease-associated genes and variants. Nucleic acids research 45:D833-D839. doi:10.1093/nar/gkw943

43. Das J, Yu H (2012) HINT: High-quality protein interactomes and their applications in understanding human disease. BMC systems biology 6:92. doi:10.1186/1752-0509-6-92

44. Shannon P, Markiel A, Ozier O, Baliga N, Wang J, Ramage D, Amin N, Schwikowski B, Ideker T (2003) Cytoscape: a software environment for integrated models of biomolecular interaction networks. Genome research 13 (11):2498-2504. doi:10.1101/gr.1239303

45. von Mering C, Jensen L, Snel B, Hooper S, Krupp M, Foglierini M, Jouffre N, Huynen M, Bork P (2005) STRING: known and predicted protein-protein associations, integrated and transferred across organisms. Nucleic acids research 33:D433-437. doi:10.1093/nar/gki005

46. Zhao J, Yang J, Tian S, Zhang W (2019) A survey of web resources and tools for the study of TCM network pharmacology. Quantitative Biology 7 (1):17-29. doi:10.1007/s40484-019-0167-8

47. Zhao J, Lv C, Wu Q, Zeng H, Guo X, Yang J, Tian S, Zhang W (2019) Computational systems pharmacology reveals an antiplatelet and neuroprotective mechanism of Deng-Zhan-Xi-Xin injection in the treatment of ischemic stroke. Pharmacol Res 147:104365. doi:10.1016/j.phrs.2019.104365

48. Janku F, Yap TA, Meric-Bernstam F (2018) Targeting the PI3K pathway in cancer: are we making headway? Nat Rev Clin Oncol 15 (5):273-291. doi:10.1038/nrclinonc.2018.28

49. Yang Q, Jiang W, Hou $P$ (2019) Emerging role of PI3K/AKT in tumor-related epigenetic regulation. Semin Cancer Biol 59:112-124. doi:10.1016/j.semcancer.2019.04.001

50. Hoxhaj G, Manning BD (2020) The PI3K-AKT network at the interface of oncogenic signalling and cancer metabolism. Nat Rev Cancer 20 (2):74-88. doi:10.1038/s41568-019-0216-7

51. Wang C, Chi Y, Li J, Miao Y, Li S, Su W, Jia S, Chen Z, Du S, Zhang X, Zhou Y, Wu W, Zhu M, Wang Z, Yang H, Xu G, Wang S, Yang J, Guan Y (2014) FAM3A activates PI3K p110a/Akt signaling to 
ameliorate hepatic gluconeogenesis and lipogenesis. Hepatology (Baltimore, Md) 59 (5):1779-1790. doi:10.1002/hep.26945

52. Quan X, Liang C, Sun M, Zhang L, Li X (2019) Overexpression of steroid receptor coactivators alleviates hyperglycemia-induced endothelial cell injury in rats through activating the PI3K/Akt pathway. Acta pharmacologica Sinica 40 (5):648-657. doi:10.1038/s41401-018-0109-4

53. Park S, Lee D, Kim D, Lee M, In G, Han S, Kim S, Lee M, Kim O, Lee J (2020) in vitroThe non-saponin fraction of Korean Red Ginseng (KGC05P0) decreases glucose uptake and transport and modulates glucose production via down-regulation of the PI3K/AKT pathway. Journal of ginseng research 44 (2):362-372. doi:10.1016/j.jgr.2019.12.004

54. Bozic M, Caus M, Rodrigues-Diez R, Pedraza N, Ruiz-Ortega M, Garí E, Gallel P, Panadés M, Martinez A, Fernández E, Valdivielso J (2020) Protective role of renal proximal tubular alpha-synuclein in the pathogenesis of kidney fibrosis. Nature communications 11 (1):1943. doi:10.1038/s41467-02015732-9

55. Edeling M, Ragi G, Huang S, Pavenstädt $H$, Susztak K (2016) Developmental signalling pathways in renal fibrosis: the roles of Notch, Wnt and Hedgehog. Nature reviews Nephrology 12 (7):426-439. doi:10.1038/nrneph.2016.54

56. Tan RJ, Zhou D, Zhou L, Liu Y (2014) Wnt/beta-catenin signaling and kidney fibrosis. Kidney Int Suppl (2011) 4 (1):84-90. doi:10.1038/kisup.2014.16

57. Zhou L, Liu Y (2015) Wnt/ $\beta$-catenin signalling and podocyte dysfunction in proteinuric kidney disease. Nature reviews Nephrology 11 (9):535-545. doi:10.1038/nrneph.2015.88

58. Duan Y, Qiu Y, Huang X, Dai C, Yang J, He W (2020) Deletion of FHL2 in fibroblasts attenuates fibroblasts activation and kidney fibrosis via restraining TGF-beta1-induced Wnt/beta-catenin signaling. J Mol Med (Berl) 98 (2):291-307. doi:10.1007/s00109-019-01870-1

59. Tharaux P, Huber T (2012) How many ways can a podocyte die? Seminars in nephrology 32 (4):394404. doi:10.1016/j.semnephrol.2012.06.011

60. Zhou L, Li Y, He W, Zhou D, Tan RJ, Nie J, Hou FF, Liu Y (2015) Mutual antagonism of Wilms' tumor 1 and beta-catenin dictates podocyte health and disease. J Am Soc Nephrol 26 (3):677-691. doi:10.1681/ASN.2013101067

61. Guo Q, Zhong W, Duan A, Sun G, Cui W, Zhuang X, Liu L (2019) Protective or deleterious role of Wnt/beta-catenin signaling in diabetic nephropathy: An unresolved issue. Pharmacological research 144:151-157. doi:10.1016/j.phrs.2019.03.022

62. Che X, Deng X, Xie K, Wang Q, Yan J, Shao X, Ni Z, Ying L (2019) Long noncoding RNA MEG3 suppresses podocyte injury in diabetic nephropathy by inactivating Wnt/ $\beta$-catenin signaling. PeerJ 7:e8016. doi:10.7717/peerj.8016

63. Hu H, Juvekar A, Lyssiotis C, Lien E, Albeck J, Oh D, Varma G, Hung Y, Ullas S, Lauring J, Seth P, Lundquist M, Tolan D, Grant A, Needleman D, Asara J, Cantley L, Wulf G (2016) Phosphoinositide 3Kinase Regulates Glycolysis through Mobilization of Aldolase from the Actin Cytoskeleton. Cell 164 (3):433-446. doi:10.1016/j.cell.2015.12.042

Page 13/19 
64. Lay A, Coward R (2018) The Evolving Importance of Insulin Signaling in Podocyte Health and Disease. Frontiers in endocrinology 9:693. doi:10.3389/fendo.2018.00693

65. Mitrofanova A, Sosa M, Fornoni A (2019) Lipid mediators of insulin signaling in diabetic kidney disease. American journal of physiology Renal physiology 317 (5):F1241-F1252. doi:10.1152/ajprenal.00379.2019

66. Sadi G, Şahin G, Bostancı A (2018) Modulation of Renal Insulin Signaling Pathway and Antioxidant Enzymes with Streptozotocin-Induced Diabetes: Effects of Resveratrol. Medicina (Kaunas, Lithuania) 55 (1). doi:10.3390/medicina55010003

67. Amato A, Santos G, Neves FA (2008) Thyroid hormone action in chronic kidney disease. Current opinion in endocrinology, diabetes, and obesity 15 (5):459-465.

doi:10.1097/MED.0b013e32830eb85e

68. Cotoi L, Borcan F, Sporea I, Amzar D, Schiller O, Schiller A, Dehelean CA, Pop GN, Borlea A, Stoian D (2020) Thyroid Pathology in End-Stage Renal Disease Patients on Hemodialysis. Diagnostics (Basel) 10 (4). doi:10.3390/diagnostics 10040245

69. Chang TT, Chen JW (2020) The Role of Chemokines and Chemokine Receptors in Diabetic Nephropathy. Int J Mol Sci 21 (9). doi:10.3390/ijms21093172

\section{Figures}

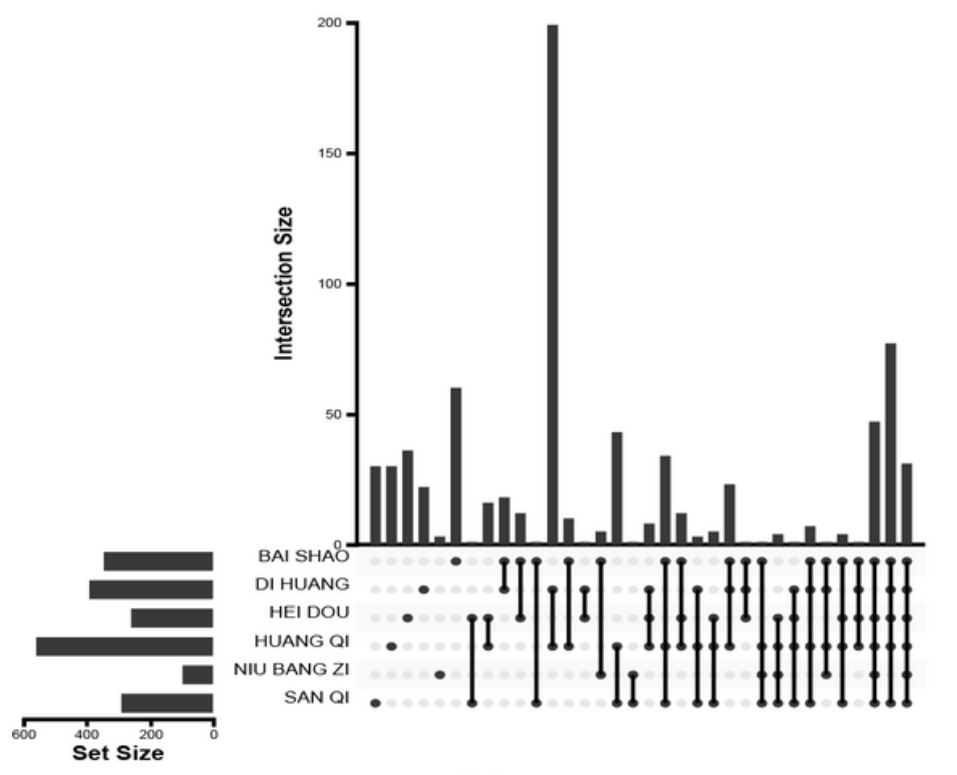

(A)

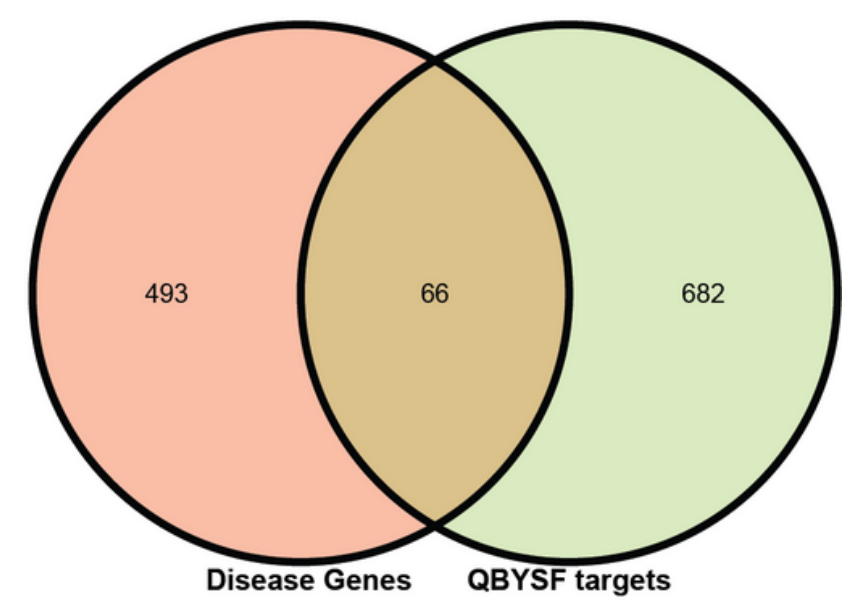

(B)

\section{Figure 1}

QBYSF target analysis. (A) The intersection between potential targets of the six herbs in QBYSF. (B) The intersection between potential QBYSF targets and diabetic kidney disease genes. 


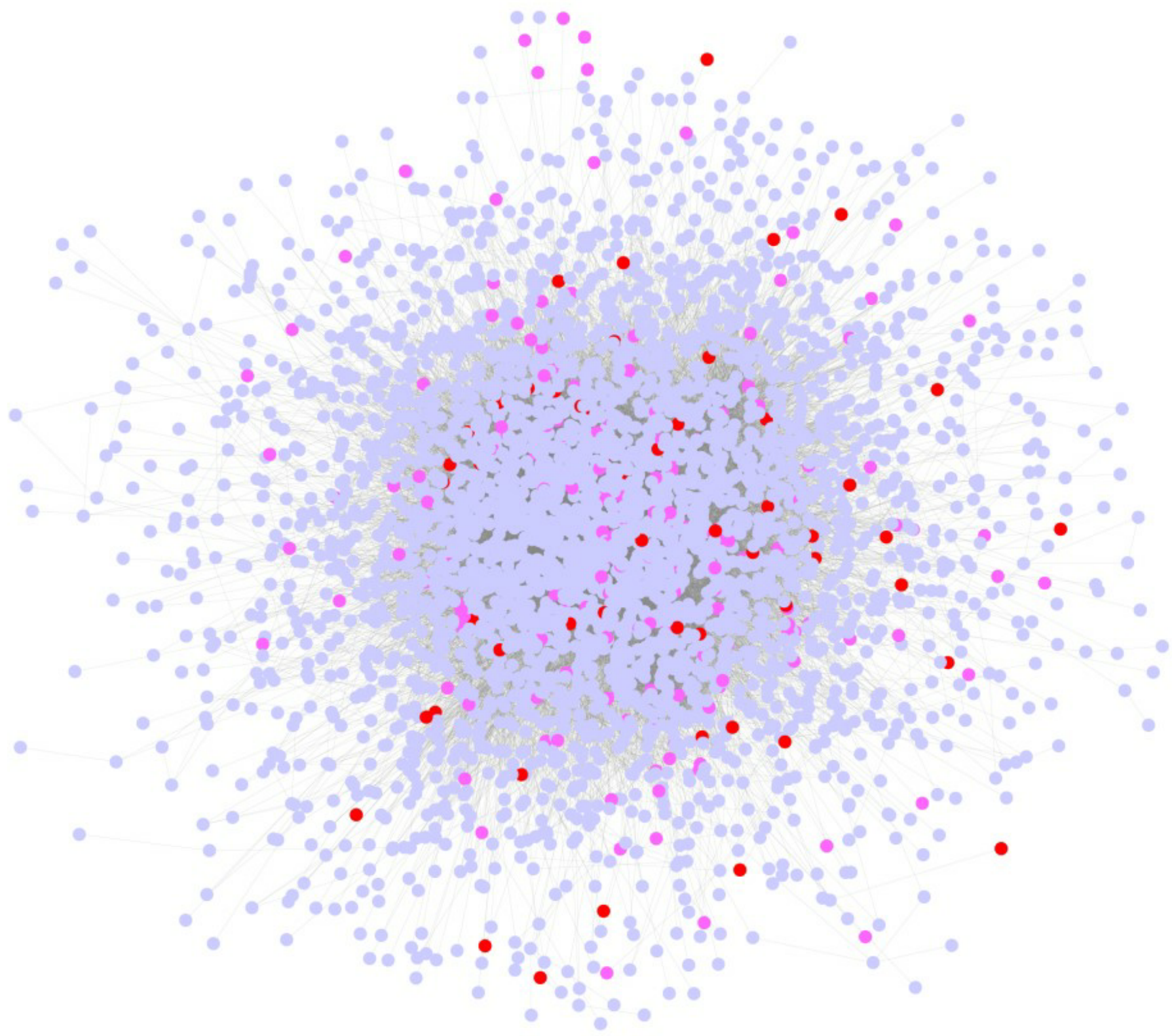

Figure 2

The diabetic kidney disease sub-network. Red and pink nodes are QBYSF targets, and red nodes are related to diabetic kidney disease genes and target proteins. 
(A)

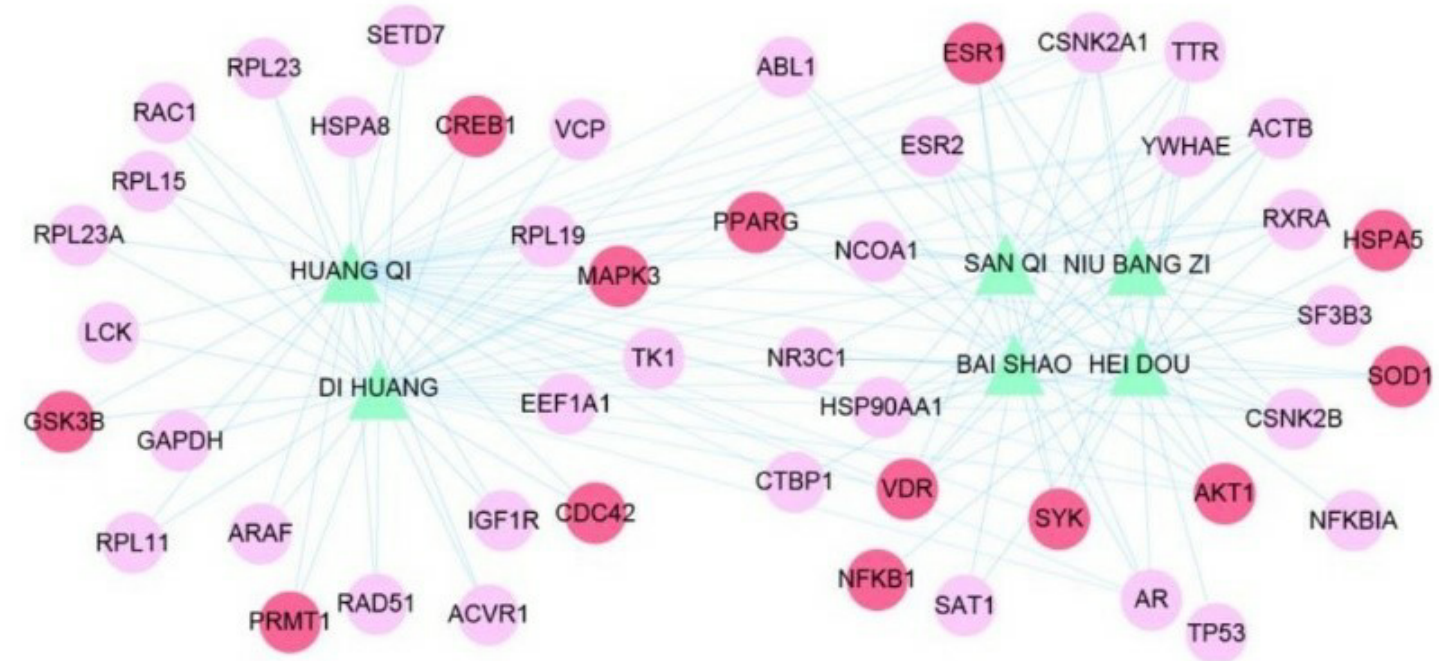

(B)

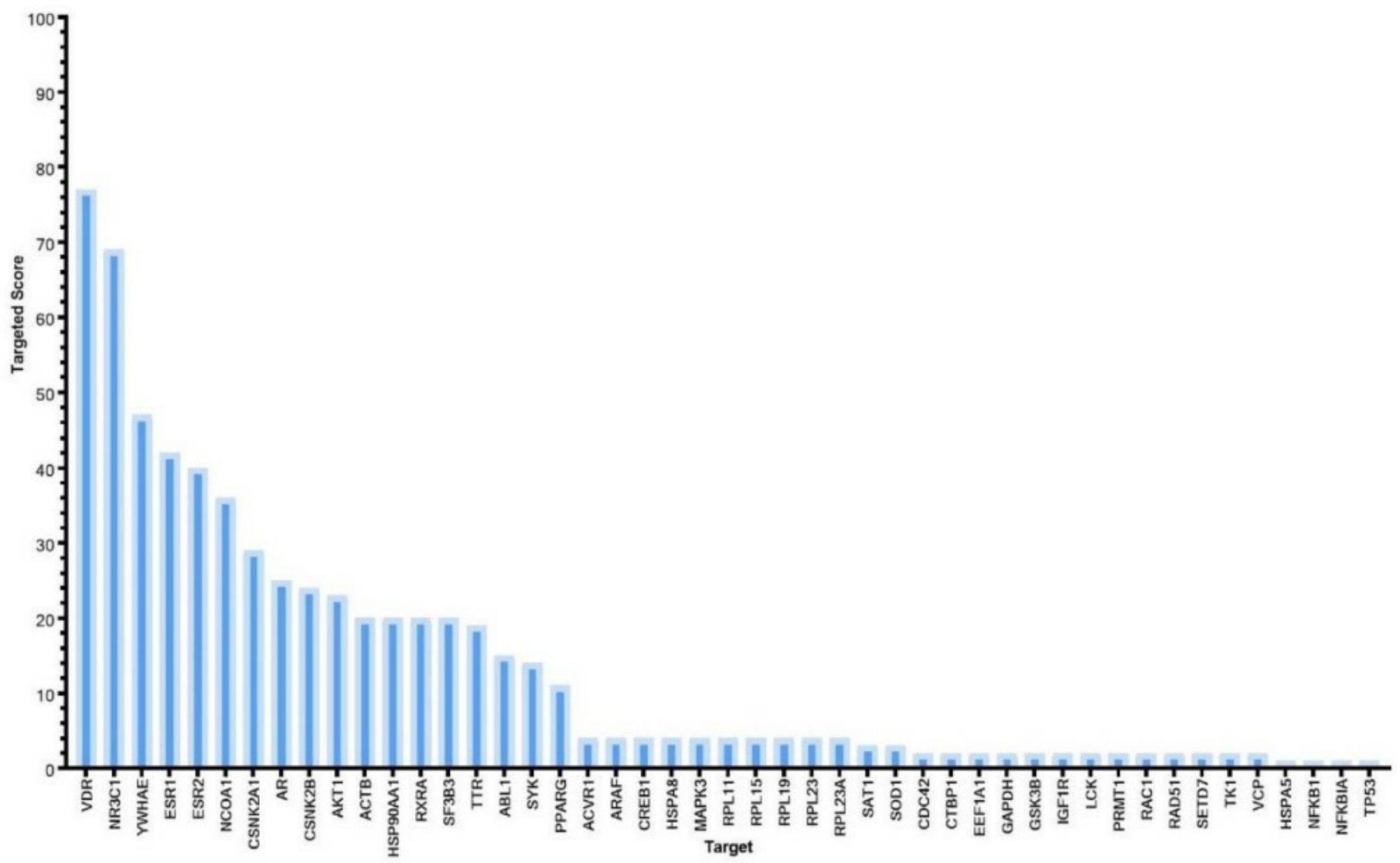

Figure 3

QBYSF targets. (A) Association network between QBYSF ingredient herbs (triangles) and QBYSF targets (circles). Red nodes are related to diabetic kidney disease. (B) Target scores for diabetic kidney diseaserelated genes. 


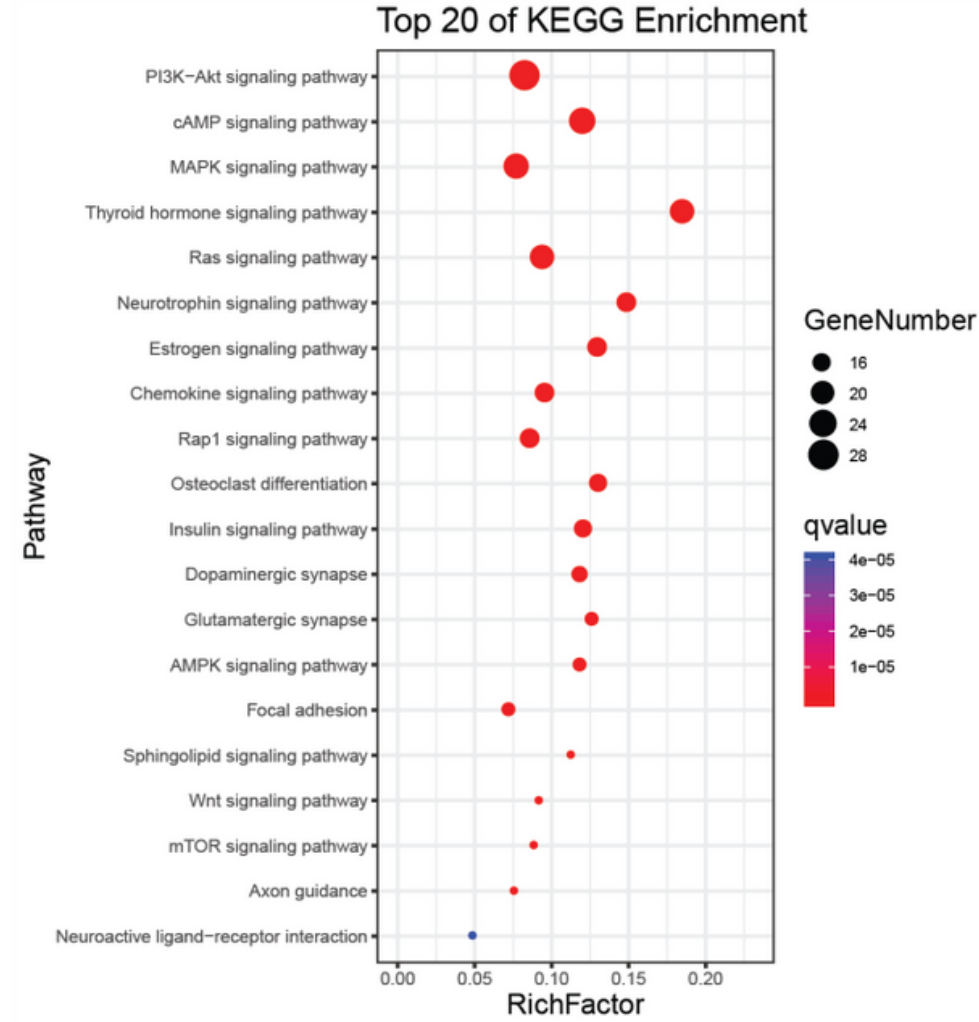

(A)

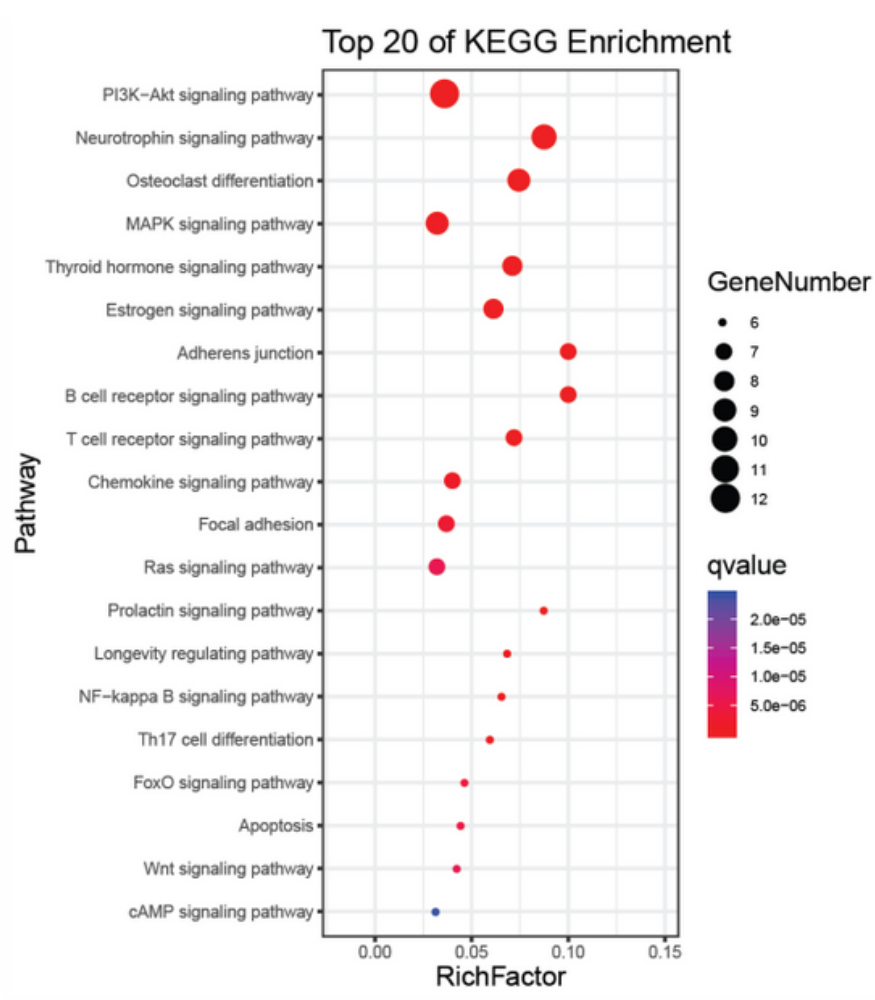

(B)

\section{Figure 4}

KEGG signaling pathways regulated by QBYSF. (A) Signal pathways regulated by the 219 targets included in the diabetic kidney disease sub-network. (B) Signal pathways regulated by the 47 hub-node targets. 


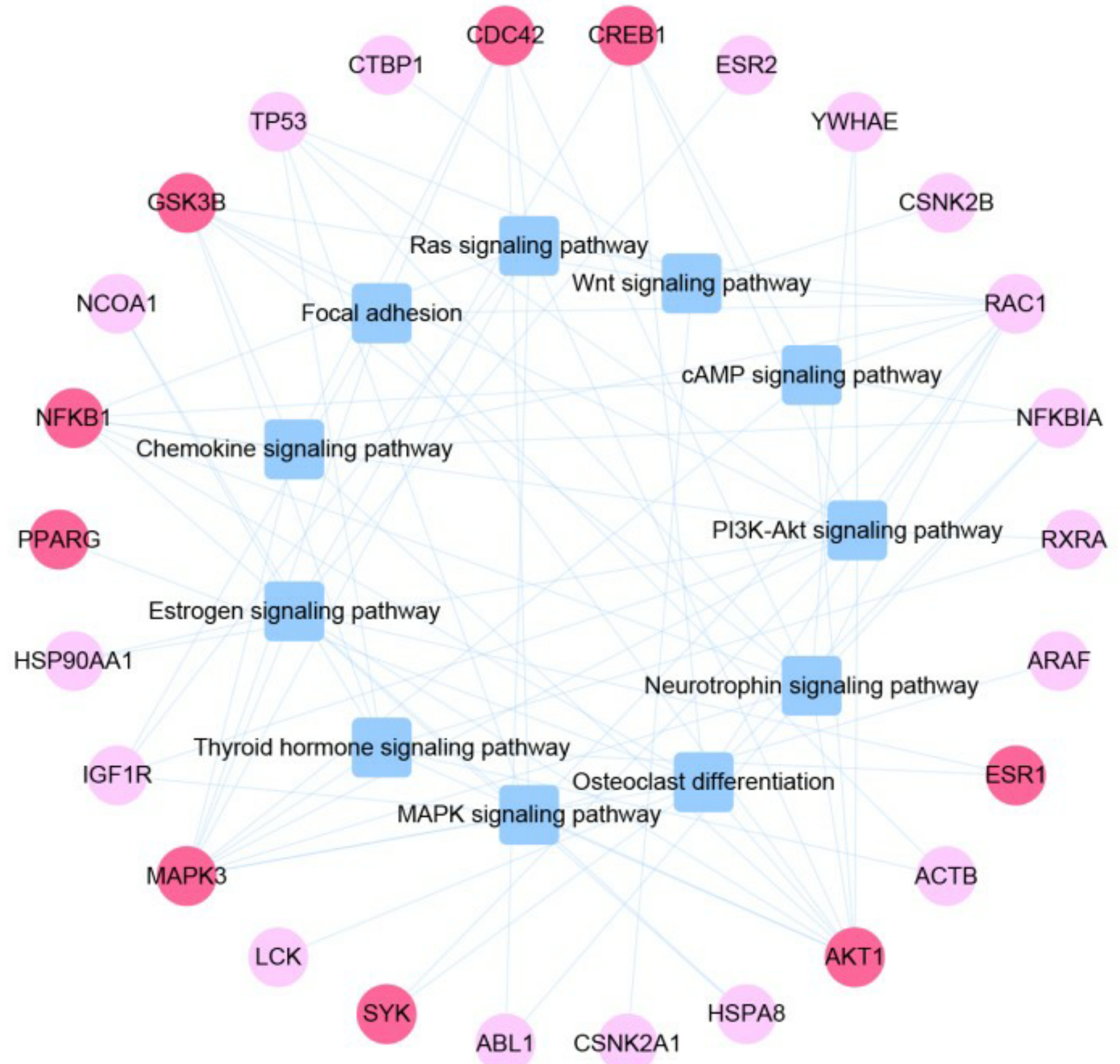

Figure 5

The association between QBYSF hub-node targets (circles) and signal pathways (boxes). Red nodes are genes related to diabetic kidney disease.

\section{Supplementary Files}

This is a list of supplementary files associated with this preprint. Click to download.

- GraphicAbstract.docx 
- SupplementaryTable1.xIsx

- SupplementaryTable2.xlsx

- SupplementaryTable3.xIsx 